\title{
Non-Rigid Shape-from-Motion for Isometric Surfaces using Infinitesimal Planarity
}

\section{Ajad Chhatkuli}

Daniel Pizarro

\section{Adrien Bartoli}

http://isit.u-clermont1.fr/ ab
ALCoV-ISIT, UMR 6284 CNRS / Université d'Auvergne, Clermont-Ferrand, France
Introduction. Non-Rigid Shape-from-Motion (NRSfM) is the general solution to the 3D reconstruction from multiple monocular images of deforming objects. Most previous attempts in NRSfM have been on learning a low dimensional shape basis from a set of contiguous images. NRSfM is very much related to the Shape-from-Template (SfT) problem, where shape is computed from a known 3D template and a single input image after deformation. Most SfT methods have been based on isometric deformations $[1,2]$. Thus applying NRSfM in isometrically constrained deformations is a natural way forward. However, there has been a gap in the literature regarding the theory behind isometric NRSfM. Many of the isometric NRSfM solutions also have practical problems. Apart from that, most of the recent works in NRSfM are based on orthographic camera models. [3] uses the orthographic camera to recover the shape's normal locally; they suffer from local two-fold ambiguities and significantly degrade for shorter focal lengths. [5] recently solved the same problem for an orthographic and perspective camera. [4] specifically addresses the case of piecewise planar surfaces; it uses the perspective camera but still has patch-wise two-fold unresolved ambiguities induced by the processing of image pairs.

In the paper, we present a general framework to solve Non-Rigid Shape-from-Motion (NRSfM) with the perspective camera for isometric deformations. Isometry allows solving for complex shape deformations from a sparse set of images. First, we formulate isometric NRSfM as a system of first-order Partial Differential Equations (PDE) involving the shape's depth and normal field and an unknown template. Second, we show the system cannot be locally resolved as such. Third, we introduce the concept of infinitesimal planarity and show that it makes the system locally solvable for three or more views. Finally, we derive an analytical solution which involves convex, linear least-squares optimization only, outperforming existing work on challenging datasets.

Modeling. We present our parametrization as in figure 2. Here, given a collection of surfaces related by isometric deformations we concentrate on two: $S_{i}$ and $S_{j}$. The embedding functions going from the unknown flat template $\mathcal{T}$ to the $3 \mathrm{D}$ surfaces are $\varphi_{i}$ and $\varphi_{j}$ respectively. $\xi_{i}$ and $\xi_{j}$ are the normal fields on the surfaces $S_{i}$ and $S_{j} . \eta_{i}$ and $\eta_{j}$ are the image warps from $\mathcal{T}$ and $\eta_{i, j}$ is the image to image warp from the image of the surface $S_{i}$ to that of $S_{j}$. Finally we represent the Jacobian of any function $\theta$ as $\mathbf{J}_{\theta}$.

NRSfM as a system of PDEs and its solvability. In the paper we describe the SfT problem and how we can go from SfT to NRSfM by recognizing that the 3D template and its flattening are unknown in NRSfM. This leads to the following, which we refer to as the NRSfM problem:

$$
\text { Find } \mid \begin{aligned}
& \mathcal{T} \subset \mathbb{R}^{2} \\
& \varphi_{i} \in C^{2}\left(\mathcal{T} ; \mathbb{R}^{3}\right) \\
& i=1, \ldots, n
\end{aligned} \quad \text { st } \quad\left\{\begin{array}{l}
\eta_{i, j}=\eta_{j} \circ \eta_{i}^{-1} \quad j=1, \ldots, n \quad j \neq i \\
\eta_{i}=\Pi \circ \varphi_{i} \\
\left(\mathbf{J}_{\varphi_{i}} \lambda \xi_{i}\right)\left(\mathbf{J}_{\varphi_{i}} \lambda \xi_{i}\right)^{\top}=\lambda^{2} \mathbf{I}_{3 \times 3}
\end{array}\right.
$$

System (1) has three constraints on the right. The first is the consistency constraint and describes how the inter-image warp is related to the image warps (from the unknown template to the images). The second is the reprojection constraint, which simply says that the image is obtained from a perspective projection of the surface embedding $\varphi_{i}$. Finally the third describes the deformation constraint for isometry. Here, we suppose the unknown flat template $\mathcal{T}$ is obtained using a conformal flattening with some unknown scaling function $\lambda$.

System (1) is analyzed in the paper to reveal that it has effectively 6 equations with 8 unknowns for 2 views. Similarly for $n$ views, we obtain $3 n$ equations with $3 n+2$ unknowns. This summarizes our second

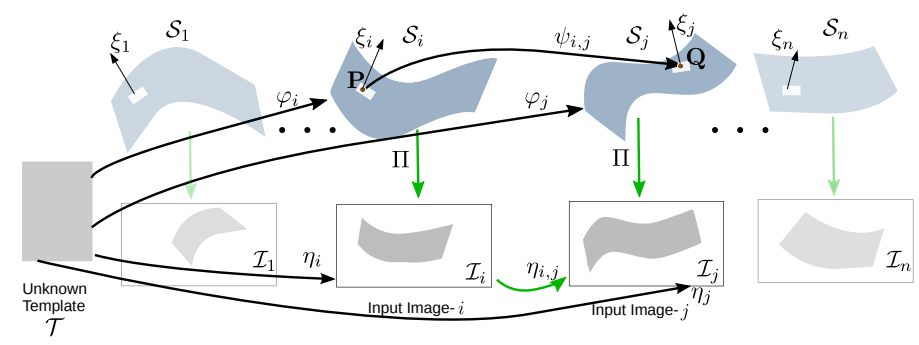

Figure 1: Geometric modeling of NRSfM.

result that one cannot solve isometric NRSfM by relaxing the relationship between the depth and the normal.

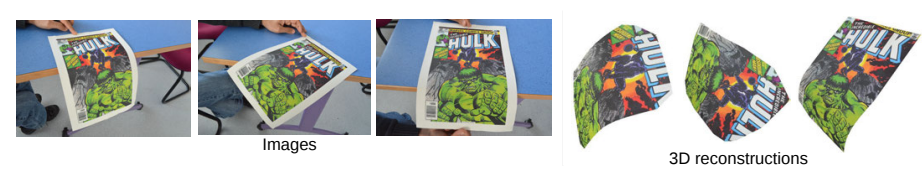

Figure 2: Images and their corresponding 3D reconstructions using our method.

Infinitesimal planarity and the solution to NRSfM. To obtain a solution to the NRSfM problem described by system (1), we assume that the surface is locally planar with zero second and higher-order derivatives. By doing this we show how isometry implies that the tangent planes on the surfaces are actually related by rigid transforms. Thus their images in turn are locally related by homographies. This is a very important result as it allows us to instead compute the aforementioned local homography to solve NRSfM. Given a known global inter-image warp we demonstrate a way to compute exactly such local homographies.

Homographies are well-understood in the literature and they can be decomposed to get the normals on the respective planes in 3D. However, there exists a two-fold ambiguity in computing the normal of a plane from its related homography. In the paper we present an algorithm to obtain the correct surface normal using at least 3 views that also robustly handles higher number of views. We tested our method with synthetic and real datasets. The results of these experiments show that our method outperforms the state of the art and is able to give a good 3D reconstruction under wide baseline viewpoints with significant deformations. We conclude that an algebraic solution of isometric NRSfM is not possible without further assumptions. However the use of infinitesimal planarity gives useful $3 \mathrm{D}$ reconstructions and is thus a valid assumption.

[1] A. Bartoli, Y. Gerard, F. Chadebecq, and T. Collins. On templatebased reconstruction from a single view: Analytical solutions and proofs of well-posedness for developable, isometric and conformal surfaces. In $C V P R, 2012$.

[2] M. Salzmann and P. Fua. Linear local models for monocular reconstruction of deformable surfaces. IEEE Trans. on Pattern Analysis and Machine Intelligence, 33(5):931-944, 2011.

[3] J. Taylor, A. D. Jepson, and K. N. Kutulakos. Non-rigid structure from locally-rigid motion. In CVPR, 2010.

[4] A. Varol, M. Salzmann, E. Tola, and P. Fua. Template-free monocular reconstruction of deformable surfaces. In CVPR, 2009.

[5] S. Vicente and L. Agapito. Soft inextensibility constraints for template-free non-rigid reconstruction. In ECCV, 2012. 